\title{
Synaesthesia lost and found: two cases of person- and music-colour synaesthesia
}

\author{
Francesca R. Farina,${ }^{1}$ Kevin J. Mitchell ${ }^{2, *}$ (D) and Richard A. P. Roche ${ }^{3, *}$ \\ ${ }^{1}$ School of Psychology, University College Dublin, Dublin, Ireland \\ ${ }^{2}$ Trinity College Institute of Neuroscience and Smurfit Institute of Genetics, University of Dublin, Trinity College, Dublin 2, Ireland \\ ${ }^{3}$ Department of Psychology, Maynooth University, Maynooth, Co Kildare, Ireland
}

Keywords: aura, conscious experience, injury, pharmacology, suppression

Edited by Sophie Molholm

Received 12 September 2016, revised 17 November 2016, accepted 23 November 2016

\begin{abstract}
Synaesthesia is a developmental condition involving cross-communication between sensory modalities or substreams whereby an inducer (e.g. a sound) automatically evokes a concurrent percept in another modality (e.g. a colour). Whether this condition arises due to atypical structural connectivity (e.g., between normally unconnected cortical areas) or altered neurochemistry remains a central question. We report the exceptional cases of two synaesthetes - subjects $A B$ and $C D$ - both of whom experience coloured auras around individuals, as well as coloured perceptions in response to music. Both subjects have, in recent years, suffered a complete loss or reduction of their synaesthetic experiences, one $(A B)$ through successive head traumas, including a lightning strike, followed by a number of medications, and the other (CD) while taking anxiolytic medications. Using semi-structured interviews and data from the Synaesthesia Battery and a colourpicker task, we characterize the phenomenological characteristics of their pre-loss synaesthesia, as well as the subsequent restoration of each subject's synaesthetic experiences (in the months post-trauma for $\mathrm{AB}$, and after cessation of medication for $\mathrm{CD}$ ). Even after years of suppression, the patterns of associations were highly consistent with those experienced pre-injury. The phenomenological experience of synaesthesia can, thus, like most conscious experiences, be modulated by pharmacologically diverse medications or head injury. However, the underlying neural substrates mediating specific synaesthetic pairings appear remarkably 'hard-wired' and can persist over very long periods even under conditions that alter or completely suppress the conscious synaesthetic experience itself.
\end{abstract}

\section{Introduction}

Synaesthetic experiences occur in $2-4 \%$ of the population (Simner et al., 2006). To date, over 60 different forms of synaesthesia have been identified (Day, 2015), while new varieties of the condition continue to be added. The most common - and most studied - form is grapheme-colour, in which colours are evoked by achromatic text (Chiou \& Rich, 2014). Sound-colour synaesthesia, whereby a sound (musical or non-musical) elicits a specific colour, is also well-documented in the literature (Rizzo \& Eslinger, 1989; Goller et al., 2009), although prevalence estimates are considerably lower $(0.2 \%$; Banissy et al., 2009). Most recently, Ramachandran et al. (2013) characterized a novel form of emotion-evoked synaesthesia involving coloured halos around faces in a subject TK. This constituted only the eighth published report of this experience, following an initial report of a 7 year-old synaesthete in 1934 (Riggs \& Karwoski, 1934), and later subjects BB (Cytowic, 1989), GW (Ward, 2004), R, F, L and M (Milán et al., 2007, 2012).

Correspondence: Kevin J. Mitchell, as above.

E-mail: Kevin.Mitchell@tcd.ie

*Joint senior authors.
Neurobiological models of synaesthesia postulate that synaesthetic experiences arise due to aberrations in neural connectivity or neural communication. Hubbard (2007) describes four neural models of the synaesthetic experience which attribute the phenomenon to cross-activation between adjacent cortical regions (due to decreased pruning of prenatal pathways), disinhibited long-range feedback in multisensory areas, feedback in re-entrant processing circuits in visual areas and beyond, and/or overactivation of parietally driven binding mechanisms (hyperbinding). Bargary \& Mitchell (2008) also argue for structural differences as a parsimonious explanation, which can be reconciled with a role for learning in the emergence of specific associations (Newell \& Mitchell (2016)). Neuroimaging studies have not distinguished between these models. Depsite many positive findings, a meta-analysis found no consistent structural differences at the macroscopic scale or consistent patterns of functional differences between the brains of synaesthetes and non-synaesthetes (Hupé \& Dojat (2015)).

Here, we report the cases of two synaesthetes - subjects $A B$ and $\mathrm{CD}$ - both of whom experience coloured auras for individuals, as well as coloured perceptions in response to music. Remarkably, both $\mathrm{AB}$ and $\mathrm{CD}$ have, in recent years, suffered a temporary loss or reduction of their synaesthetic experiences due to head trauma and 
medication, respectively. We believe this constitutes the first report of the loss and subsequent return of these forms of synaesthetic experiences, and suggest that the study of such rare cases may shed light on the nature of this phenomenon.

\section{Results}

Synaesthete AB, a 21 year-old ambidextrous (left-hand dominant) female, has had synaesthetic perceptions since childhood. Specifically, she experiences projected visual colours in response to musical notes, chords and instruments, and strong associated colours or auras in response to people. Her musical concurrents are unidirectional, and appear - typically in the centre of the visual field - as semi-transparent percepts comparable to ink or paint dropped into water. Evoked colours are temporally synchronous to the on/offset of the sounds, and are influenced by characteristics such as pitch (higher notes present higher in the visual field with more pastel shades; lower notes appear in lower regions with more solid colours), volume (louder evokes more intense colour), type of instrument (see Fig. 1a for colours associated with different notes before

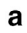

\section{C\# D D\# E B F F\# G G\# A A\# High C C C\#D D\#E B F F\# G G\# A A\#High C}

b
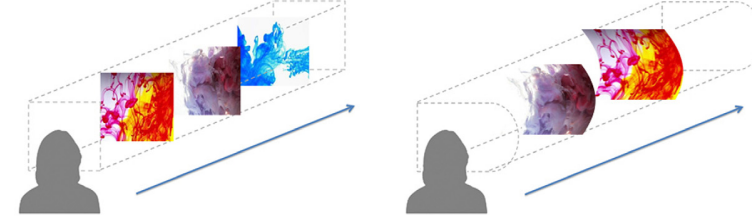

c

Powerful, energetic, passionate Scientific, detail-orientated Balanced, caring, nature-loving Loyal, empathic, trustworthy Intuitive, emotional, selfless Original, outgoing, open-minded Optimistic, intelligent, inspiring

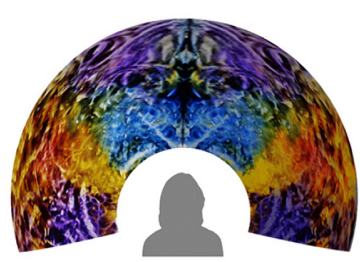

d

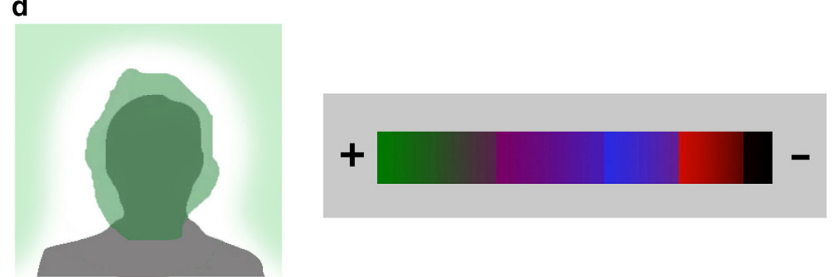

FIG. 1. (a) RGB-accurate colour concurrents for musical notes for $A B$ before (upper) and after (lower) loss of synaesthetic experiences. Consistency pre- and post-loss, calculated via correlation coefficients: Red: 0.71, Green: 0.74, Blue: 0.63 . Mean $=0.69$. (b) Depiction of AB's experience of music from piano (left) and flute (right) wherein colour percepts are experienced in the centre of the visual field within the confines of an imaginary box/corridor. The shape of the corridor's boundaries varies according to the instrument; e.g. for the flute, it appears 'more curvy to the right side'. (c) Left: RGB-accurate colour concurrents for AB's personality traits associated with people; Right: depiction of AB's experience of a complete song (Flight Facilities - Stand Still) whereby colour concurrents are described 'as if projected on the inside of a dome' above her head. (d) Left: depiction of CD's coloured auras, with independent central and peripheral concurrents; Right: RGB-accurate colour concurrents for CD's emotions, ranging from positive associations (green, purple) to ambiguous (blue, red) to negative (brown, black). Colour block sizes reflect the frequency of occurrence of each emotional association in CD's experience. [Colour figure can be viewed at wileyonlinelibrary.com]. and after loss, and Fig. $1 \mathrm{~b}$ for representations of different instruments) and expectation (more vivid colours for unexpected sounds). Despite being unable to read sheet music, AB can play the tin whistle (a traditional Irish flute), flute, glockenspiel, marimba and piano by ear, and cites her learning these instruments as being aided by her synaesthesia, where 'wrong' colours flag out of tune notes (see Fig. 1c, right, for a representation of a complete song). Her personcolour associations are not projected in the visual field; rather, known individuals evoke a strong colour association in the mind's eye, with specific personality traits linked to different colours (e.g. blue people are emotional, green are loyal; see Fig. 1c, left, for a list). No two people have the same colour, but colours for couples can intermingle; further, some people can have multiple colours, while a person's voice can differ from the colour of their personality. Colours are strongly influenced by emotion, and $\mathrm{AB}$ again states that this form of synaesthesia is beneficial in that her emotional response to a person is partly determined by their colour.

Between the ages of 13 and 16, $\mathrm{AB}$ suffered two minor concussions, neither of which affected her synaesthetic experiences (see Fig. 2). These traumas did, however, lead to increased migraines for which she prescribed Paramax (paracetamol with metoclopramide hydrochloride) at age 16 . While on this medication, AB experienced a short-lived suppression of her coloured concurrents. $\mathrm{AB}$ started at university at age 18 , at which time she was experiencing synaesthetic associations as normal. While at university, AB was introduced to the condition of synaesthesia, prompting her to complete the online Synesthesia Battery (Eagleman et al., 2007; see Table 1). In the battery, synaesthetes are indicated by a score below one and non-synaesthetes by a score of two or above. AB scored below one on the piano scale-colour (0.57) and instrument-colour scales (0.78). She did not receive a chord-colour score as her colours were not sufficiently varied to make a valid assessment. As part of the battery, $\mathrm{AB}$ completed the Vividness of Visual Imagery Questionnaire (VVIQ-2), a widely used measure of self-reported imagery (Marks, 1973). Individuals who score 3 or above are considered to visualize more strongly than the general population; $\mathrm{AB}$ scored above average (4.72). She also completed the projector-associator measure included in the battery. 'Projector' and 'associator' categories are used to describe the way in which an individual's synaesthesia is experienced. Projectors physically see their synaesthetic associations in space (e.g. the colour red projected onto the letter A printed in black), while associators experience their associations in the mind's eye, i.e. they are aware that the letter A is red, but do not physically see this colour (Dixon et al., 2004). AB's score was negative $(-1)$, indicating a projector-type association. She also reported having personality-colour and emotion-colour associations, although these were not assessed in the battery.

At age 19, $\mathrm{AB}$ contracted viral meningitis, resulting in a change in her experienced colours for the duration of the illness (one month); they remained vivid, but were evoked by the 'wrong' notes and appeared 'displaced'. AB did not experience any other sensory disturbances during this time. After her recovery, she reports that the colours returned to normal. In the months that followed, she sustained two further concussions. The first of these caused her to lose consciousness; $\mathrm{AB}$ reports that following this incident, her musicevoked colours moved from the centre to the lower periphery of her visual field and appeared muted or softened, without much change to her person-colour synaesthesia. The second concussion produced no loss of consciousness, but resulted in increased anxiety and emotionality, and a change in her synaesthetic experiences. These became quite intense and vibrant, and also bothersome as the colours 'seemed weird' and easily led to sensory overload. Again, the 

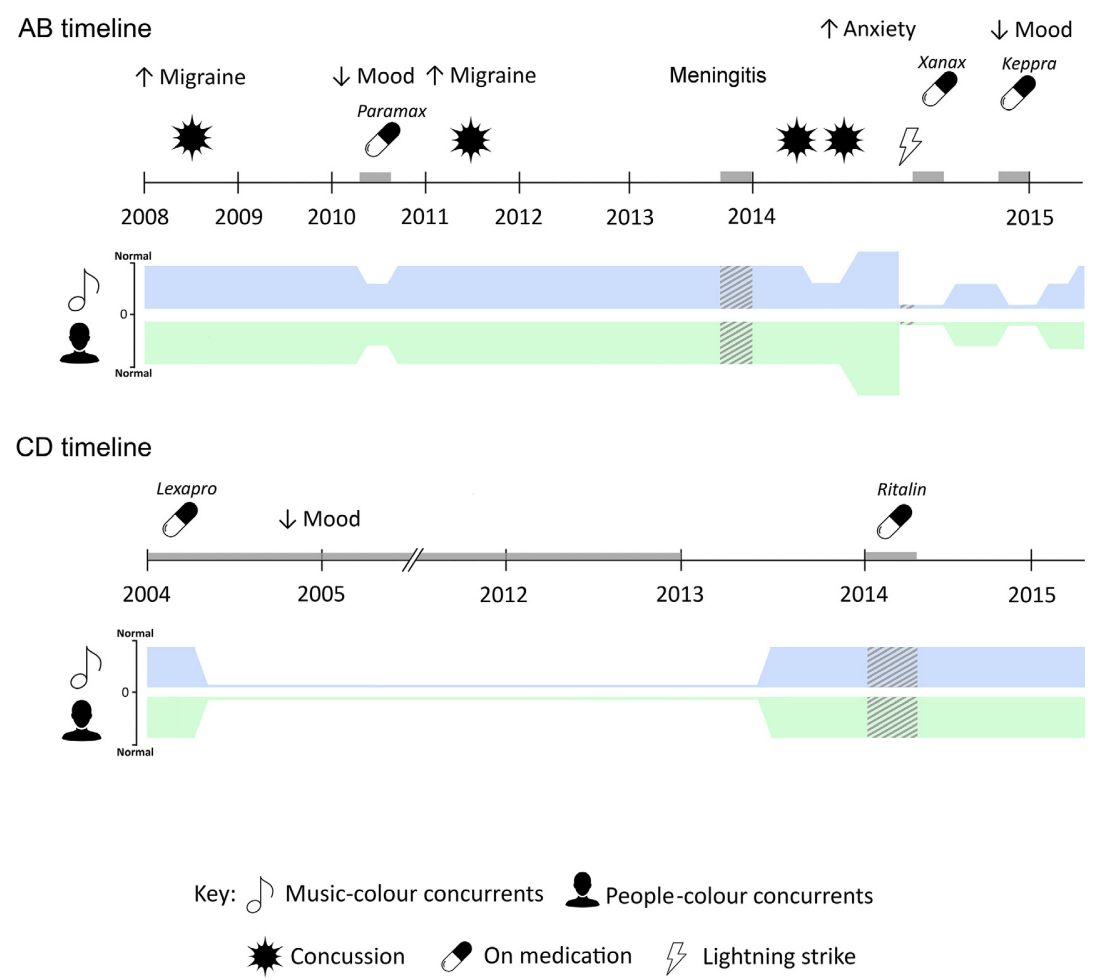

FIG. 2. Timeline of events and resultant effects on synaesthetic experience for AB (upper) and CD (lower). Significant events depicted include concussions/ head trauma, migraine, seizures, taking medication and lightning strike. Grey blocks indicate time spent on medication, or duration of meningitis. Hashed areas on colour bars indicate periods of altered synaesthetic colours. Icons were sourced from thenounproject.com and include Lightning Bolt by artworkbean, Pill by Sergey Demushkin, Burst by Bohdan Burmich, User by JM Waideaswaran and Music by Sherrinford. [Colour figure can be viewed at wileyonlinelibrary.com].

TABle 1. Data from the Online Synesthesia Battery (Eagleman et al., 2007) for AB (taken at ages 18 and 20) and CD (age 31). For inducer-concurrent pairs, scores below 1.0 (in bold) indicate the presence of synaesthesia; for visual imagery, a Vividness of Visual Imagery Questionnaire (VVIQ-2) score above 3.0 indicated above average imagery. Negative scores on the projector-associator scale are indicative of associator-type

\begin{tabular}{lll}
\hline & Age 18 & Age 20 \\
\hline Subject AB & & \\
Battery Results & Piano scale $\rightarrow$ colour: $\mathbf{0 . 5 7}(<1.0$ synaesthetic) & Piano scale $\rightarrow$ colour: 1.08 \\
& Instruments $\rightarrow$ colour: $\mathbf{0 . 7 8}(<1.0$ synaesthetic) & Instruments $\rightarrow$ colour: $\mathbf{0 . 4 5}$ \\
& Visual imagery: $\mathbf{4 . 7 2}(>3$ higher than average) & Visual imagery: $\mathbf{5}$ \\
& Projector associator: $-\mathbf{1}$ (negative $=$ associator) & Chord $\rightarrow$ colour: $1.84(<1.0$ synaesthetic) \\
Subject CD & & Age 31 \\
Battery Results & - & Instruments $\rightarrow$ colour: $\mathbf{0 . 5 7}(<1.0$ synaesthetic) \\
& - & Visual imagery: $\mathbf{4 . 2 2}(>3$ higher than average) \\
& - & Projector associator: $-\mathbf{0 . 3 3}$ (negative $=$ associator) \\
\hline
\end{tabular}

sensory disturbances experienced by $\mathrm{AB}$ were restricted to her synaesthesia.

Two months later, by which time her experiences were 'on their way back to normal', $\mathrm{AB}$ was involved in a lightning strike (she was inside a metal cabin with her hand on the windowsill when the structure was hit by lightning), leading to a brief hospitalization. This event had several effects, only some of which were related to her synaesthesia. $\mathrm{AB}$ experienced memory loss for a period of several days immediately after the lightning strike, as well as increased anxiety afterwards. In addition, her normal colour experiences were essentially abolished, though she did experience 'an awful lot of white' due to a ringing in her ears, and later developed pins and needles, which she describes as seeing white flashes in her head.
She suffered a number of panic attacks during this time and describes uncharacteristic synaesthetic experiences preceding them, including perceptions of intense, strange colours, mostly golds and silvers, which she describes as possibly 'not even real colours'. Following the lightning strike, $\mathrm{AB}$ was prescribed Xanax (Alprazolam) for one month. This caused a temporary muting of her colours for both music and people, but by July of that year they had partially returned to their original colours with high consistency $(r=0.69$; calculated via RGB correlation coefficients, see Fig. 1a). Over this time, $\mathrm{AB}$ developed seizures, which led to her being prescribed Keppra (Levetiracetam) in December of the same year. This resulted in a complete suppression of her synaesthetic colour experiences, as well as generally low mood and difficulty concentrating. After a 
month of increasing dosage of this medication, $\mathrm{AB}$ was removed from it due to the severe side effects. Within the 8 -year period described, $\mathrm{AB}$ was not prescribed any other medications that affected her synaesthesia. She did report occasional use of overthe-counter medication for migraines (Ibuprofen); however, this had no effect on her synaesthetic experiences. She also reported a single instance of recreational drug use (cannabis). This caused her to experience heightened emotionality which temporarily enhanced her synaesthesia for this period (approximately $4 \mathrm{~h}$ ), similar to the effects of a seizure.

By the following year, $\mathrm{AB}$ had returned to normal cognitive and emotional functioning, but reports no restoration of her evoked colours at that time. Increased migraine caused heightened emotionality and distractibility, along with a partial return of her concurrents, albeit with altered or 'wrong' colours. Normal colour associations returned one month later. In June, by which time she was seizurefree, $\mathrm{AB}$ completed a second Synesthesia Battery (see Table 1) and semi-structured interview with the authors. She scored positively for instrument-colour associations (0.45), while her scores for piano scale-colour (1.08) and chord-colour (1.84) were inconclusive (i.e. less than two). $\mathrm{AB}$ reported that she experienced different colours for each note within a given chord, and therefore found it difficult to choose one colour on these measures. As such, she was instructed to select the colour which was the strongest/most vivid of those experienced. This may explain why her colour-associations were more consistent than those observed when $\mathrm{AB}$ first completed the battery. $\mathrm{AB}$ also had an above average score of 5 on the VVIQ-2. AB's colour concurrents for people were examined using a RGB colour-picker task (similar to Eagleman et al., 2007). In this task, $\mathrm{AB}$ was presented with the names of familiar (family and friends, chosen by $\mathrm{AB}$ herself) and unfamiliar people (chosen by the experimenters and introduced to $\mathrm{AB}$ shortly before the task) and asked to adjust the colour of each person using a RGB colour-picker until it matched her synaesthetic colour (three presentations; randomized order). AB's scores were found to be highly consistent for all categories (i.e. family members, friends and strangers; see Fig. 3, Top).

$\mathrm{CD}$, a 31 year-old ambidextrous (right-hand dominant) male with a family history of autism, has experienced coloured auras around

\begin{tabular}{|c|c|c|c|}
\hline$A B$ & Presentation 1 & Presentation 2 & Presentation 3 \\
\hline \multirow{4}{*}{ Relatives } & Ellen & Ellen & Ellen \\
\hline & George & George & George \\
\hline & James & James & James \\
\hline & Oliver & Oliver & Oliver \\
\hline \multirow{4}{*}{ Friends } & Raymond & Raymond & Raymond \\
\hline & Eve & Eve & Eve \\
\hline & Linda & Linda & Linda \\
\hline & Brian & Brian & Brian \\
\hline \multirow{4}{*}{ Strangers } & Keith & Keith & Keith \\
\hline & Florence & Florence & Florence \\
\hline & David & David & David \\
\hline & Robert & Robert & Robert \\
\hline
\end{tabular}

\begin{tabular}{|c|c|c|c|c|}
\hline CD & Presentation 1 & Presentation 2 & Presentation 3 & \\
\hline \multirow{4}{*}{ Relatives } & Laura & Laura & Laura & \multirow{8}{*}{0.99} \\
\hline & Darren & Darren & Darren & \\
\hline & Emma & Emma & Emma & \\
\hline & Mark & Mark & Mark & \\
\hline \multirow{4}{*}{ Friends } & Peter & Peter & Peter & \\
\hline & Bradley & Bradley & Bradley & \\
\hline & Shane & Shane & Shane & \\
\hline & Teresa & Teresa & Teresa & \\
\hline \multirow{4}{*}{ Strangers } & Robert & Robert & Robert & \multirow{4}{*}{0.91} \\
\hline & Florence & Florence & Florence & \\
\hline & Keith & Keith & Keith & \\
\hline & Naomi & Naomi & Naomi & \\
\hline
\end{tabular}

FIG. 3. RGB-accurate colour concurrents for specific people for AB (upper) and CD (lower) on three successive occasions using a colour-picker. Consistency scores were calculated by correlating red, green and blue values for each set of names (relatives, friends, strangers) across presentations 1 and 2, 1 and 3 and 2 and 3, resulting in a correlation coefficient for each colour for each category. These three colour coefficients were then averaged to produce mean correlation scores for each name type. Mean correlation coefficients for red, green and blue values are shown for each category: Relatives/Family Members; Friends; Strangers/Unknown. Names included in the Relatives and Friends categories were self-selected by AB and CD. Names in the Strangers/Unknown category were selected by the authors; these individuals were introduced briefly to $\mathrm{AB}$ and $\mathrm{CD}$ before the task. All names have been changed to preserve anonymity. [Colour figure can be viewed at wileyonlinelibrary.com]. 
people and colours in response to music since childhood. Colours for people are automatically experienced around the person (usually the head) in the visual field, with specific colours associated with CD's mood and/or emotional reactions to the person (see Fig. 1d). The degree to which auras are projected in space depends largely on the colour itself; for example, brown and black are typically perceived in sustained projections, while red appears much less frequently. When hearing music, he perceives semi-independent 'colour blotches' in the centre and periphery of the visual field; unlike $\mathrm{AB}$, these are entirely based on his emotional reactions to the music. He also has vague but consistent colour associations for some numbers, and visualizes numeric functions in three-dimensional space; this can be used as an arithmetical aid. CD also possesses mild but consistent emotional associations for some letter groupings (e.g. 'abc' and 'ijk'), as well as personification of letters (although these personalities tend to be inconsistent and were more prevalent during his childhood). Up to the age of $30, \mathrm{CD}$ viewed his synaesthetic experiences as being profoundly negative.

At age 20, while taking Lexapro (Escitalopram) for Seasonal Affective Disorder, he first experienced a reduction of his visualizations, with a complete loss of colours for people and music occurring within 4-5 weeks. This absence persisted until the age of 27, when he ceased taking the medication, resulting in the eventual return in June of that year of coloured concurrents to people and music (Fig. 2). CD was briefly prescribed Ritalin (Methylphenidate) the following January, leading to an alteration of his perceived colours for people; specifically, perceived colours were more purpletinted and/or purple auras were enhanced. CD was not prescribed any other drugs during this time. By the time he completed the Synesthesia Battery (see Table 1) and semi-structured interview (age 30 ), CD's original perceptual experiences (which he now views as positive) were returning and his visualizations were 'becoming more coherent'. His battery scores indicated an associator-type synaesthete $(-0.33)$ with instrument-colour associations $(0.57)$ and above average VVIQ (4.22). CD's person-colour associations were found to be highly consistent across categories (see Fig. 3, Bottom).

\section{Discussion}

Both of these cases of music- and person-colour synaesthesia follow the classic descriptions of developmental synaesthesia, rather than being injury- or drug-induced. Yet, the synaesthetic experiences were modified by injury, by infection, migraine or seizure, or by a variety of drugs. Whether synaesthesia arises due to atypical structural connectivity (e.g., between normally unconnected cortical areas) or altered neurochemistry remains a central question. These results provide some supporting evidence for the neuroanatomical account; specifically, the changes to AB's synaesthetic experiences during seizures and migraines (akin to a recent case study by Alstadhaug \& Benjaminsen, 2010), and following head trauma. However, AB also experienced non-synaesthetic sensorimotor disturbances in some cases, e.g. after the lightning strike. Thus, we cannot exclude the possibility that the synaesthetic changes observed were mediated by other, unknown effects occurring as the result of these incidents.

It is also possible to interpret the drug effects reported here as supporting the neurochemical account of synaesthesia, though the diversity of pathways involved suggests another interpretation. Previous reports on the pharmacology of synaesthesia have focused mainly on the range of hallucinogenic drugs that can induce audiovisual synaesthesia-like experiences in non-synaesthetes or enhance them in synaesthetes, with a smaller number of reports of drugs that modulate the experience of developmental synaesthesia (see Sinke et al., 2012; for a recent review). Several authors have noted that many of these drugs, most notably the hallucinogens LSD, psilocybin and mescaline, act by modulating serotonin signalling (see Luke \& Terhune, 2013) even leading to the proposal that mutations in serotonin pathway genes might underlie the condition (Brang \& Ramachandran, 2008). Lexapro, which completely suppressed the synaesthetic experiences of $\mathrm{CD}$ for the 8 years he was taking it, is a selective serotonin reuptake inhibitor, which is consistent with previous reported effects of fluoxetine (Brang \& Ramachandran, 2008; Luke et al., 2012). However, the other drug effects reported here do not support such a selective relationship to serotonin signalling. Metoclopromide (present in Paramax) is an antagonist of dopamine receptors; Levetiracetam (Keppra) binds synaptic vesicle glycoprotein SV2A and modulates presynaptic L-type calcium channels, leading to reduced neurotransmitter release; Alprazolam (Xanax) is a benzodiazepine which allosterically increases chloride flux through GABA-A receptors, thus increasing inhibition; and Methylphenidate (Ritalin) is a norepinephrine and dopamine reuptake inhibitor. Thus, whatever the neural substrate of these synaesthetic experiences, they can clearly be modulated by a diverse range of drugs targeting very different pathways. In this respect, synaesthesia does not differ from most other conscious experiences. The pattern of drug responsiveness does not implicate a particular neurochemical pathway, nor does it suggest that synaesthetic experiences arise from a disturbance in such a pathway.

Remarkably, the drugs and other factors described here did not just change the intensity of the synaesthetic experience or affect whether it reached conscious awareness. They also led to qualitative changes in the nature of the concurrent percepts, specifically in the location in space of projected colours or in the specific colours induced. Furthermore, the changes were not short-lived - they persisted over days, weeks, months or years. However, despite the series of unfortunate events which caused successive alterations to AB's synaesthetic experiences over an 8-year period and the complete suppression of CD's synaesthesia also over an 8-year period, in both cases their synaesthesia has since returned to a similar level and qualitative nature as prior to these events. In the case of $\mathrm{AB}$, direct comparison of colours for musical notes before and after the most severe set of events (recorded at ages and 18 and 21; Fig. 2a) shows a number of changes but an overall high level of consistency (0.69). These findings provide strong evidence that the neural substrates of synaesthetic associations, once they are consolidated in what is presumably an early critical period (Newell \& Mitchell, 2016), remain 'hard-wired' thereafter and can persist over very long periods even under conditions that alter or completely suppress the conscious synaesthetic experience itself.

\section{Acknowledgements}

The authors gratefully acknowledge the contributions of Julia Januszweski and Laura Rai (Maynooth University) for transcribing the interviews with $\mathrm{AB}$ and $\mathrm{CD}$, and Ríona McArdle (University of Newcastle) for the initial introduction to $\mathrm{AB}$. Special thanks to $\mathrm{AB}$ and $\mathrm{CD}$ for their time, openness and willingness to engage with our tasks, and for feedback on the manuscript. Ethical approval for data collected was granted by the Maynooth University Research Ethics Committee, BSRESC-2015-001.

\section{References}

Alstadhaug, K.B. \& Benjaminsen, E. (2010) Synesthesia and migraine: case report. BMC Neurol., 10, 1.

Banissy, M.J., Kadosh, R.C., Maus, G.W., Walsh, V. \& Ward, J. (2009) Prevalence, characteristics and a neurocognitive model of mirror-touch synaesthesia. Exp. Brain Res., 198, 261-272.

Bargary, G. \& Mitchell, K.J. (2008) Synaesthesia and cortical connectivity. Trends Neurosci., 31, 335-342. 
Brang, D. \& Ramachandran, V.S. (2008) Psychopharmacology of synesthesia: the role of serotonin $\mathrm{S} 2 \mathrm{a}$ receptor activation. Med. Hypotheses, $\mathbf{7 0}$, 903-904.

Chiou, R. \& Rich, A.N. (2014) The role of conceptual knowledge in understanding synaesthesia: evaluating contemporary findings from a "hub-andspokes" perspective. Front. Psychol., 5, 1-55.

Cytowic, R.E. (1989) Synaesthesia: a Union of the Senses. Springer, New York.

Day, S.A. (2015) Linguistic Synesthesia. In Wright, J.D. (Ed.), International Encyclopedia of the Social \& Behavioral Sciences, 2nd Edn. Elsevier, Amsterdam, 193-198.

Dixon, M.J., Smilek, D. \& Merikle, P.M. (2004) Not all synaesthetes are created equal: projector versus associator synaesthetes. Cogn. Affect. Behav. Neurosci., 4, 335-343.

Eagleman, D.M., Kagan, A.D., Nelson, S.S., Sagaram, D. \& Sarma, A.K. (2007) A standardized test battery for the study of synesthesia. J. Neurosci. Meth., 159, 139-145.

Goller, A.I., Otten, L.J. \& Ward, J. (2009) Seeing sounds and hearing colors: an event-related potential study of auditory-visual synesthesia. J. Cognitive Neurosci., 21, 1869-1881.

Hubbard, E.M. (2007) Neurophysiology of synesthesia. Curr. Psychiatry Rep., 9, 193-199.

Hupé, J.M. \& Dojat, M. (2015) A critical review of the neuroimaging literature on synesthesia. Front. Human Neurosci., 9, 1-35.

Luke, D.P. \& Terhune, D.B. (2013) The induction of synaesthesia with chemical agents: a systematic review. Front. Psychol., 4, 1-12.

Luke, D., Terhune, D.B. \& Friday, R. (2012) Psychedelic synaesthesia: evidence for a serotonergic role in synaesthesia. Seeing Perceiving, 25, 74
Marks, D.F. (1973) Visual imagery differences in the recall of pictures. Brit. J. Psychol., 64, 17-24.

Milán, E.G., Hochel, M., González, A., Tornay, F., McKenney, K., Díaz Caviedes, R., Mata Martín, J.L., Rodríguez Artacho, M.A. et al. (2007) Experimental study of phantom colors in a color blind synaesthete. J. Consciousness Studies, 14, 75-95.

Milán, E.G., Iborra, O., Hochel, M., Rodríguez Artacho, M.A., DelgadoPastor, L.C., Salazar, E. \& González-Hernández, A. (2012) Auras in mysticism and synaesthesia: a comparison. Conscious. Cogn., 21, 258268.

Newell, F.N. \& Mitchell, K.J. (2016) Multisensory integration and crossmodal learning in synaesthesia: a unifying model. Neuropsychologia, 88, $140-150$.

Ramachandran, V.S., Miller, L., Livingstone, M.S. \& Brang, D. (2013) Colored halos around faces and emotion-evoked colors: A new form of synesthesia. Neurocase, 18, 352-358.

Riggs, L.A. \& Karwoski, T. (1934) Synaesthetsia. Br. J. Psychol., 25, 2941.

Rizzo, M. \& Eslinger, P.J. (1989) Colored hearing synesthesia An investigation of neural factors. Neurology, 39, 781 .

Simner, J., Mulvenna, C., Sagiv, N., Tsakanikos, E., Witherby, S.A., Fraser, C., Scott, K. \& Ward, J. (2006) Synaesthesia: the prevalence of atypical cross-modal experiences. Perception, 35, 1024-1033.

Sinke, C., Halpern, J.H., Zedler, M., Neufeld, J., Emrich, H.M. \& Passie, T. (2012) Genuine and drug-induced synesthesia: a comparison. Conscious. Cogn., 21, 1419-1434.

Ward, J. (2004) Emotionally mediated synaesthesia. Cognitive Neuropsych., 21, 761-772. 